2013-02

\title{
Visuospatial memory in dyslexia: Evidence for strategic deficits
}

\author{
Bacon, Alison
}

http://hdl.handle.net/10026.1/2930

10.1080/09658211.2012.718789

Memory

Informa UK Limited

All content in PEARL is protected by copyright law. Author manuscripts are made available in accordance with publisher policies. Please cite only the published version using the details provided on the item record or document. In the absence of an open licence (e.g. Creative Commons), permissions for further reuse of content should be sought from the publisher or author. 
VISUOSPATIAL MEMORY IN DYSLEXIA: EVIDENCE FOR STRATEGIC DEFICITS

\author{
Alison M. Bacon ${ }^{1}$, Fabrice B. R. Parmentier ${ }^{2,3}$ \& Polly Barr ${ }^{1}$ \\ ${ }^{1}$ Plymouth University, UK \\ ${ }^{2}$ University of the Balearic Islands, Palma \\ ${ }^{3}$ University of Western Australia
}

Corresponding author:

Dr Alison M. Bacon

School of Psychology

Plymouth University

Drake Circus

Plymouth PL4 8AA

Tel: 01752584805

Fax: 01752584808

Email: ambacon@plymouth.ac.uk 


\section{Abstract}

Impairments in working memory are suggested to be one of the defining characteristics of dyslexia and deficits in verbal recall are well documented. However, the situation regarding visuospatial memory is less clear. In a widely used measure, the Corsi blocks task, sequences of visuospatial locations can be recalled forwards, in the order presented $(\mathrm{CF})$ or backwards, in reverse order $(\mathrm{CB})$. Previous research has suggested that while $\mathrm{CF}$ draws on spatialsequential resources, CB may load executive and distinctly visual processes. While dyslexics typically show no deficit on $\mathrm{CF}, \mathrm{CB}$ is rarely presented. We present three studies which indicate a consistent dyslexic deficit on $\mathrm{CB}$ that can be ameliorated by visual strategy instructions. Our data suggest that, without instruction, dyslexics are unable to adopt an effective CB strategy and this is consistent with a deficit in executive function. These results have implications for our understanding of visuospatial memory in dyslexia, and also in terms of the administration of the Corsi task to special populations. 
Acknowledgements: this research was supported by the UK Economic \& Social Research Council grant no. RES-000-22-2815 awarded to Alison Bacon and Fabrice Parmentier. The authors would also like to thank Professor Jackie Andrade for her helpful comments on earlier drafts of this paper. 
Developmental dyslexia is typically characterised by a difficulty in mastering key phonological skills such as fluent and accurate reading, word comprehension, spelling and writing. Dyslexia may also present a spectrum of impairments affecting a range of other cognitive functions including (but not limited to) planning, organisation of information, attention and memory (e.g., Miles, 1993; Nicolson \& Fawcett, 2001). The deficits are incommensurate with intelligence, unrelated to socioeconomic status, and a strong genetic link has been identified (for reviews see Démonet, Taylor, \& Chaix, 2004; Vellutino, Fletcher, Snowling \& Scanlon, 2004). The present research focused on working memory function in adults with dyslexia. The perseveration of dyslexia related difficulties throughout the lifespan can have an impact on achievement in education and employment as well as many informal aspects of everyday life (McLoughlin, Fitzgibbon \& Young, 1994; Morgan \& Klein, 2000; Smith-Spark, Fawcett, Nicolson \& Fisk, 2004). Impairments in working memory have been described as one of the major defining characteristics of dyslexia (McLoughlin, et al, 1994) and in the studies reported here we investigate a population for whom working memory ability has clear implications for success, university students. For these individuals, their dyslexia may not simply affect the ability to remember information, but also underpin the automaticity of a range of study skills including learning from lectures, note taking, producing coursework assignments and examination answers and practicalities such as using the library or mastering keyboard skills (e.g., Mortimore \& Crozier, 2006).

Deficits in working memory can be conceptualised in terms of a tripartite model (e.g., Baddeley \& Hitch, 1974; Baddeley \& Logie, 1999) comprising a central executive (CE), responsible for controlling and manipulating information stored in two slave subsystems, the phonological loop (PL) dealing with verbal information, and the visuospatial sketchpad 
(VSSP) for visuospatial material. These are considered to be relatively short-term storage systems within which to-be-remembered information is maintained by a process of verbal rehearsal in the PL or spatial eye tracking in the VSSP (see Baddeley, 2007 for a recent account of the WM model). Research has consistently demonstrated that dyslexics are impaired in short-term memory for verbal information, a process which plays an implicit role in reading and spelling (e.g., Ackerman \& Dykeman, 1993; Engle, Cantor \& Carullo, 1992; Jeffries \& Everatt, 2004; Kibby, Marks, Morgan \& Long, 2004; Martinez Perez, Majerus, Mahot \& Poncelet, 2012; Miles, 1993; Vellutino, 1979). Such difficulties are well documented in studies employing simple measures of verbal storage designed to draw on PL resources (e.g. Pickering, 2006; Plaza, Cohen, \& Chevrie-Muller, 2002; Tijms, 2004; Wagner, \& Muse, 2006). One such measure is the digit span task, in which participants are asked to recall digits presented in series of increasing length and their verbal memory span is defined as the maximum sequence length recalled correctly. A low verbal span is a common marker of dyslexia and versions of this test are frequently used as part of dyslexia assessment (McLoughlin, et al., 1994). Furthermore, deficits are also observed on complex verbal span tasks which measure working memory (WM) - the capacity to store information while concurrently engaging in other cognitively demanding activities such as the manipulation or longer-term retention of information. Children with dyslexia are consistently found to present deficits on verbal WM tasks relative to typically developing controls (Siegel \& Ryan, 1989; Swanson, 1994, 1999; Swanson, Ashbaker, \& Lee, 1996). Such tasks implicate the PL, but also draw significantly on the CE. Rather than being a unitary resource, the CE is thought to comprise separate components for domain-specific storage and domain-general processing and individual differences in performance on complex WM tasks reflect capacity in these resources (Bayliss, Jarrold, Gunn \& Baddeley, 2003). In dyslexia, verbal WM span scores are found to predict reading ability independently of measures of verbal STM (e.g., Swanson, 
2003; Swanson \& Howell, 2001; Swanson \& Jerman, 2007) further suggesting deficits in executive processes. Recent neurophysiological studies present converging evidence suggesting abnormalities in brain activation (Beneventi, et al, 2010) and connectivity (Wolf, et al, 2010) during verbal WM tasks.

While verbal memory function has been researched extensively in dyslexia, the visuospatial domain has received relatively little attention and the extant evidence is equivocal. In contrast to the verbal domain, dyslexics typically show no impairment in visuospatial short-term memory (e.g., Brosnan, Demetre, Hamill, Robson, Shepherd and Cody, 2002; Gould \& Glencross, 1990; Jeffries \& Everatt, 2004; Kibby, Marks, Morgan \& Long, 2004; Palmer, 2000) and it is frequently presumed that verbal memory deficits do not extend to the visuospatial domain. Prevalent and influential claims of enhanced visuospatial abilities in dyslexia (e.g., Aaron \& Guillemond, 1993; Davis, 1997; Galaburda, 1993; Vail, 1990; West, $1997 \& 2008$ ) may be one reason why the notion that visuospatial memory remains intact has been so readily accepted. However, consistent with the verbal domain, deficits are observed on complex span tasks which measure visuospatial WM. If dyslexics are not impaired in short-term visuospatial storage (VSSP capacity) then deficits in executive processing are likely to explain their performance on these complex WM tasks (e.g., Reiter, Tucha \& Lange, 2004; Smith-Spark \& Fisk, 2007). A recent meta-analysis (Swanson, Zheng \& Jerman, 2009) concludes that while there is consistent evidence for a significant relationship between verbal WM and reading level, dyslexic performance on visuospatial tasks appears to fluctuate with processing demands. In other words, individuals with reading disabilities such as dyslexia have most difficulty with tasks which place particularly high demands on both storage and maintenance components of the working memory system, implicating the CE. 
The three studies we present here are concerned with further understanding the processes which underlie visuospatial memory performance in dyslexia. We employ the Corsi Blocks Task (CBT; Corsi, 1972), a measure widely used in research and clinical settings (for review see Berch, 1998). The CBT is often considered a visuospatial equivalent of the verbal digit span and, as such, is designed to measure serial recall of visuospatial information. In its traditional manual form, the CBT apparatus consists of an irregular arrangement of nine blocks fixed to a flat board. The administrator taps the blocks in randomized sequences of increasing length and the participant attempts to recall and reproduce each sequence of tapped locations. Visuospatial short-term memory span is defined as the maximum sequence length that the participant is able to reproduce. In more recent research, computerised versions have been administered whereby the apparatus is replaced by an on-screen array of squares which are highlighted in sequence to mimic the process of physical tapping.

Studies which have drawn on Corsi data to suggest that the VSSP is intact in dyslexia have tended to present only the forwards recall version of the task (CF), where participants are asked to recall sequences of locations in the order originally presented (e.g., Gould \& Glencross, 1990; Jeffries \& Everatt, 2004; Palmer, 2000). However, there is growing evidence that performance on the backwards recall version $(\mathrm{CB}$; where sequences of locations are recalled in reverse order) may draw on functions other than short-term VSSP storage capacity. In the verbal domain, research has consistently shown that recalling digit sequences in reverse order results in worse performance than when they are recalled in a forward direction. This is observed in neurotypical populations as well as in dyslexia, and is generally attributed to the additional executive demands required to transform the sequence into reverse order (e.g., Schofield \& Ashman, 1986). An association between executive and visuospatial functions is well established in the working memory literature (e.g., Miyake et 
al., 2001) and a concurrent task of spatial tapping (designed to inhibit VSSP storage) interferes more with performance on $\mathrm{CF}$ than $\mathrm{CB}$, further suggesting that while forward recall draws on visuospatial resources, backwards recall in the CBT may also draw on executive processes and these may compensate for spatial interference (e.g., Berch et al., 1998; Kemps, 1999, 2001; Vecci \& Richardson, 2001). Given the evidence for executive deficits in dyslexia, this makes $\mathrm{CB}$ an interesting subject of study.

Vandierendonck, Kemps, Fastame \& Szmalec (2004) systematically tested CBT performance under a range of differing secondary task conditions designed to interfere selectively with visuospatial, verbal or executive processing. Their results indicated that visuospatial, but not verbal, processes were implicated in $\mathrm{CF}$ as might be expected. However, with longer sequences or $\mathrm{CB}$, where the cognitive load is greater, additional executive resources were implicated. Furthermore, in $\mathrm{CB}$, they present evidence for distinctly visual (as opposed to spatial) representations whereby locations are represented as a static simultaneous pattern, a path linking the to-be-remembered locations allowing the participant to visually bind information about location and order (see also Vandierendonck \& Szmalec, 2004).

This suggestion is interesting in the light of evidence that that visuospatial memory can be fractionated into distinct and separable visual and spatial subsystems (e.g., Della Sala, Gray, Baddeley, Allamano, \& Wilson, 1999; Logie, 1995; Logie \& Pearson, 1997; Pickering, 2001). The dissociation is supported by numerous neurological studies that indicate independence of brain activation to visual and spatial stimuli (e.g. Courtney, et al, 1996; Smith et al, 1996) or selective impairment following brain injury (e.g. Carlesimo et al, 2001; Wilson et al, 1999). Furthermore, visual and spatial memory functions show fractionable developmental trajectories. Logie and Pearson (1997) administered a static visual pattern 
recall task and a Corsi type task (forwards recall) to children aged between 5 and 12 . Performance increased with age for both tasks, however the age-related increase was more pronounced for the static-visual task, suggesting that the visual and spatial subcomponents of the VSSP were distinct and develop at different rates. Pickering, Gathercole, Hall, and Lloyd (2001) presented similar tasks, controlling for visuospatial content while varying static or dynamic presentation formats. Their results supported Logie and Pearson's suggestion of developmental fractionation and, in addition, showed that the individual involvement of the two VSSP subcomponents was not dependent on the actual visuospatial characteristics of the to-be-recalled stimuli, rather upon whether that stimulus was static or dynamic in nature. In other words, the separable visual and spatial subsystems appear to be sensitive to static and dynamic features of the stimuli, respectively. Della Sala et al (1997) have developed a span measure of specifically visual short-term memory for static patterns, the Visual Patterns Test (VPT). They report a series of studies with both healthy and brain-lesion participants which indicated a separation between visual and sequential capacities in the VSSP, with the VPT and CBT drawing on discrete subcomponents. Della Sala et al (1999) showed that distinctively spatial and visual secondary tasks have differential effects on the two measures, with visual interference disrupting VPT performance and spatial interference disrupting recall on $\mathrm{CF}$.

Overall, if both $\mathrm{CF}$ and $\mathrm{CB}$ are presented, the CBT provides a useful tool for investigating distinctly visual, spatial and executive memory function in dyslexia. The evidence above suggests that backwards CBT recall may draw upon CE resources, and also on the distinctly visual subcomponent of VSSP if a static visual pattern of locations is recalled. The standard CF task (where dyslexics typically show no deficit) is likely to draw more strongly on the spatial subcomponent of VSSP if the locations are recalled in the manner presented - i.e. as a 
dynamic sequence. However, there is some suggestion that visual recall may potentially be effective on $\mathrm{CF}$. where sequences can be maintained as visual patterns without serial order involvement because all the to-be-remembered locations are visible during sequence presentation. As such, only the path between them has to be remembered (Smyth \& Scholey, 1992; Wilde, Strauss, \& Tulsky, 2004). These accounts aim to explain why, in contrast to digit span, recall accuracy in the forward and backward CBT tends to be comparable. In line with this view, Kessels (2008) suggested that clinical populations showing a reduction of performance in the Corsi task do so to a similar degree under both forward and backward recall conditions.

This suggests that two potential strategies are available for CBT, a dynamic spatial-sequential one or a visual one based on remembering the pattern of locations and/or path between them. In dyslexia, a preference for visual strategies has been observed in other cognitive domains. For instance, in reasoning and problem solving tasks where non-dyslexic individuals use verbal processes, dyslexics have been shown to use strategies which rely heavily on visual mental representation (Bacon, Handley \& McDonald, 2007; Bacon \& Handley, 2010a). Interestingly, visual short-term memory as measured by the VPT is found to be predictive of reasoning accuracy for dyslexics, but not non-dyslexics, despite similar VPT span scores (Bacon \& Handley, 2010a; 2010b). This raises an additional question as to whether CF performance in dyslexia reflects intact dynamic-spatial memory or the ability to draw on compensatory visual resources. The three studies we report below aimed to extrapolate the processes used in both $\mathrm{CF}$ and $\mathrm{CB}$ in order to investigate the extent to which visuospatial memory function in dyslexia is as intact as has been claimed. 


\section{Experiment 1}

As there is a paucity of research comparing dyslexic and non-dyslexic individuals over both versions of the CBT, Experiment 1 presents a simple comparison of performance on both forward $(\mathrm{CF})$ and backward $(\mathrm{CB})$ recall. Evidence suggests that backward recall on the $\mathrm{CBT}$ involves executive processes and, as individuals with dyslexia exhibit executive deficits, we predicted that they would perform less well than non-dyslexics on CB. However, a comparable level of recall in both versions of the task would suggest that dyslexics are able to draw on an effective strategy for $\mathrm{CB}$, one that does not rely so heavily on the CE. Distinctly visual static memory processes are also implicated in backwards recall and dyslexics have been found to draw on such resources in a compensatory way in other cognitive domains. As such, Experiment 1 also presented the VPT in order to explore the possible role of visual processes in CBT. If participants are remembering CBT locations as a static visual pattern then we might expect to observe a significant relationship between VPT and CBT span scores.

\section{Methods}

\section{Participants}

One hundred and thirty eight students from Plymouth University took part. They were recruited via advertisements on the university student internet homepage and through an established paid participant pool. Participants were undertaking a range of undergraduate degree courses and hence all were at a broadly similar educational level. All participants were native English speakers. Dyslexic participants $(n=68,32$ males, 36 females) had all been formally diagnosed by one of a team of Chartered Educational Psychologists employed at Plymouth University. As such, all had received a recent, full-scale, diagnostic assessment and 
received a formal statement certifying their dyslexic status and indicating that their difficulties were not associated with any other co-morbid learning difficulty. Two participants reported mild dyspraxia, no other co-morbid conditions were disclosed. The non-dyslexic control group ( $\mathrm{n}=70 ; 22$ males, 48 females) self-declared as not having dyslexia or any other reading, learning or developmental disorder. Self-reports of being non-dyslexic have been found to be highly accurate (Nicolson and Fawcett, 1997). In addition, three screening tasks were administered to all participants to further validate our sampling.

Nonsense word reading (NWR). All participants read a NWR passage taken from Fawcett and Nicolson's (1998) Dyslexia Adult Screening Test (DAST). NWR is known to be impaired in dyslexia and such measures are suggested to identify deficits even in compensated dyslexics, that is, those individuals whose academic achievement has been sufficient for them to progress into higher education but who still experience considerable dyslexia related difficulties (Lefly \& Pennington, 1991; Miller-Shaul, 2005; Smith-Spark, et al., 2004). The test comprises a short written passage which the participant reads aloud. The passage tells a simple fairytale style story with a number of words substituted by complex nonsense words, for instance the passage begins with the sentence: In the olden days, a nobactious rennifer set out to craiberg an enormous dollitroy that threatened his lammersill country. In line with DAST guidelines, the score is derived from number of correctly read real and nonsense words and time taken to read the passage. The maximum score is 99 .

Spelling: The spelling sub-test from Wechsler Objective Reading Dimensions (WORD; Wechsler, 1993) was also administered. This test comprises 50 words presented in order of increasing difficulty. The words to be spelled are read out by the researcher, firstly as a single word and then within the context of a simple sentence, for instance: PATIENTS. The patients 
were waiting to see the doctor. Test score is number of correctly spelled words, maximum 50. The WORD spelling test has been used previously in this context in research into WM function in adults with dyslexia (Smith-Spark et al., 2004; Smith-Spark \& Fisk, 2007) but, as the test is only normed to age 16:11 raw scores, rather than standard scores, are reported.

Short-form IQ measure: We presented all four subtests from the Wechsler Abbreviated Scale of Intelligence (WASI; Wechsler, 1999):

Vocabulary: designed to test word knowledge and verbal concept formation. Participants are required to provide oral definitions for a set of 42 words of increasing difficulty, for example, item 9 (the starting point for adult participants) is "Bird", whereas the final item 42 is "Panacea".

Similarities: measures verbal reasoning and concept formation. A series of 26 word pairs are presented and participants asked to state how each pair is alike. The items increase in difficulty with the first being "grape and strawberry" (both fruits) and the final item being "tradition and habit" (both routine behaviour patterns). For each of these subtests, Wechsler (1999) specifies response criteria which allows for the award of 1 or 2 points per item depending on the level of difficulty and accuracy of definition. The maximum score for vocabulary is 84 and for similarities it is 48 .

Matrix Reasoning: measures visual information processing and abstract reasoning skills. 35 test items each comprise an abstract pattern or figure, from which a section is missing. The task is to identify the missing section from a selection of five possible responses. One point is awarded for each correct response hence the maximum possible score is 35 . 
Block Design: assesses abilities such as analysis and synthesis of abstract visual stimuli, nonverbal concept formation and the ability to separate figure and ground in visual stimuli. The participant uses a set of coloured blocks to replicate a series of designs previously presented by the examiner. The 13 designs progress in difficulty from those requiring two blocks to complex designs requiring nine blocks. A time limit of 60 seconds is imposed for the simpler designs, rising to 120 seconds for the nine block items. A score of between 4 and 7 is awarded for each correct design according to time taken. The total possible score is 71.

Raw scores for each subtest are converted into age-corrected T-scores. Verbal IQ (VIQ) is obtained by summing the T-scores from the two verbal subtests (vocabulary and similarities), and Performance/nonverbal IQ (PIQ) can be obtained by summing the T-scores from the two performance subtests (matrix reasoning and block design). T-scores from all four subscales can be used to obtain an age-corrected Full Scale IQ (FSIQ) score.

Appendix A presents screening data for all three experiments presented in this article. As a diagnosis of dyslexia is confirmatory of a significant deficit in literary skills in the context of a normal range IQ, we would not expect the two groups of participants to differ in terms of their WASI scores. However, if our sampling is robust, dyslexics should perform significantly less well than non-dyslexics on both spelling and NWR. As Appendix A shows, this was indeed the case which suggests that the group allocations were valid and differed only in terms of a dyslexic diagnosis. 


\section{Design and Procedures}

In a fully within-subjects design, all participants were presented with a computerised version of the Corsi blocks task under both $\mathrm{CF}$ and $\mathrm{CB}$ recall conditions. The task was presented using E-prime experimental software. Participants were presented with an onscreen array of 9 blank squares against a white background. The squares were arranged following Corsi's (1972) original array. The squares turned black for $1000 \mathrm{~ms}$, one at a time and with a stimulus-onset asynchrony of $2000 \mathrm{~ms}$, to form the to-be-remembered sequences. Sequences increased in difficulty from 2 to 9 locations, with two trials at each level. Participants completed all trials. Different sets of sequences were used in the $\mathrm{CF}$ and $\mathrm{CB}$ versions of the task. A total of ten sets of 16 sequences ( $2 \times$ list lengths 2 to 9) were prepared, out of which two were randomly selected, for each participant, to be used in the $\mathrm{CF}$ and $\mathrm{CB}$ conditions. Given past demonstrations of the impact of sequence complexity on visuospatial serial memory (Parmentier, 2011; Parmentier \& Andrés, 2006; Parmentier, Andrés, Elford, \& Jones, 2006; Parmentier, Elford, \& Maybery, 2005), the ten sets were carefully matched in terms of the length of the path formed by the to-be-remembered sequences, the number of path crossings they contained, and the mean angle they formed. Across sets, the mean path length was 528.09 pixels $(S D=242.86)$ and the mean angle was 47.58 degrees $(S D=16.93)$. All sets were identical with respect to the number of path crossings $(M=2.64, S D=2.97)$. Path length and angles varied very slightly across sets but were statistically identical, $F$ $(9,130)<1, M S E=63516, p=1$, and $F(9,130)<1, M S E=304.2, p=.99$, respectively. The order of presentation of $\mathrm{CF}$ and $\mathrm{CB}$ was counterbalanced within each participant group to control for order effects. Before beginning each main experimental task, all participants completed 6 practice trials (three sequences each of two and three locations). The span score 
was defined as the highest level at which a participant could recall at least one sequence correctly.

Following the Corsi tasks, all participants completed a computerised version of the Visual Patterns Test (VPT). In this task, participants were presented with squares forming grids of increasing sizes. In each grid, half the squares were filled in black while the remaining ones remained empty. The participants' task was to remember these patterns in order to reproduce them immediately afterwards in an empty grid. Each pattern was presented onscreen for 3 sec. Participants clicked on the to-be-remembered squares in any order they wished. As they clicked on squares, these turned black. They were allowed to unmark any previously recalled square by clicking on it again. Only when the appropriate number of squares were marked did a button appear on the screen allowing participants to initiate the next trial. Three patterns were presented for each of the 14 levels of difficulty (grids containing 2 to 15 locations marked squares). Participants completed all trials. Following Della Sala et al. (1997), the visual memory score was calculated as the mean number of filled squares correctly recalled in the last three patterns recalled entirely correctly.

\section{Results}

Figure 1 suggests a pattern of recall in line with our predictions. Non-dyslexic participants performed similarly on $\mathrm{CF}(M=6.34, S D=1.02)$ and $\mathrm{CB}(M=6.12, S D=1.21)$. Dyslexics however, perform better on $\mathrm{CF}(M=6.25, S D=1.03)$ than on $\mathrm{CB}(M=5.22, S D=.84)$. 
A 2 (group: dyslexic, non-dyslexic) x 2 (recall direction: $C F, C B$ ) repeated measures ANOVA carried out on the Corsi scores presented two significant main effects, of direction: $F(1,136)=41.28, M S E=.66, p=<.001, \eta^{2}=.23$, and of group: $F(1,136)=11.35, M S E=$ $1.48, p=.001, \eta^{2}=.08$. These effects are clearly shown in Figure 1 which illustrates that participants generally performed better on $\mathrm{CF}$ than on $\mathrm{CB}$, and that non-dyslexics performed better overall than dyslexics. Importantly, we also observed a significant interaction between group and direction: $F(1,136)=16.84, M S E=1.48, p=.001, \eta^{2}=.08$. Simple withingroups comparisons confirmed that non-dyslexics performed comparably on $\mathrm{CF}$ and $\mathrm{CB}(p>$ .3). For the dyslexics, however, CB span score was significantly lower than that observed for $\mathrm{CF}, t(67)=7.58, p<.001$. Between-group comparisons confirmed comparable CF scores $(p$ $>$.60) while non-dyslexics performed significantly better than dyslexics on CB: $t(136)=$ $5.06, p<.001$

The groups performed comparably on VPT $(p=.24$; dyslexic $M=10.81, S D=1.98$; nondyslexic $M=11.21, S D=.24)$. Examining the relationship between performance on this task and Corsi recall, we observed a significant correlation between VPT and CB scores for nondyslexic participants $(r=.43 ; p<.001)$, but a negligible relationship for dyslexics $(r=.07 ; p$ $=.58)$. The between-group difference in correlations was statistically significant $(Z=2.24, p=$ .03). There was little evidence of a relationship between VPT and CF scores for either group (dyslexics $r=.07$; non-dyslexics $r=.12 ; p>.30$ in both cases).

\section{Discussion}

The aim of this first study was to directly compare dyslexic and non-dyslexic participants on $\mathrm{CF}$ and $\mathrm{CB}$. Our results support our initial prediction in that participants with dyslexia present deficits in the backward version of the Corsi task while the groups perform comparably on 
CF. Previous research (e.g. Pickering, et al, 2001; Vandierendonck, et al, 2004) has suggested that $\mathrm{CB}$ recall involves the encoding and maintenance of locations as an static visual pattern, rather than a sequence. In Experiment 1, non-dyslexic participants presented a significant relationship between visual memory ability (VPT score) and CB recall. This suggests that those non-dyslexics with higher visual memory ability score more highly on CB. This group performed significantly better than the dyslexics who show no correlation between $\mathrm{CB}$ and VPT scores and who also do less well on CB compared to CF.

An executive deficit in dyslexia may seem to provide a straightforward explanation for this data: Dyslexic participants appear to be unable to employ a recall strategy drawing on those functions. However, our results also point to a potentially important role for visual processes in backwards recall (c.f. Vandierendonck et al., 2004). While the effective recall strategy for $\mathrm{CB}$ may draw on executive functions, it may also involve visual short term maintenance. More specifically, it may be that participants who perform best on CB (the non-dyslexics), on realising the difficulty of the task, attempt to abandon a serial spatial recall strategy and opt instead to remember the visual pattern formed by the to-be-remembered locations when envisaged simultaneously. Importantly however, this is unlikely to reflect a deficit of visual memory per se in dyslexia since both groups performed equally well on the VPT. Instead, our data suggest that it may be the adoption of an inappropriate strategy that is responsible for the dyslexics' deficit in CB.

The suggestion that dyslexics have difficulty drawing on visual memory resources when necessary is interesting given that they are known to do so in other domains, such as reasoning (Bacon, et al, 2007; Bacon \& Handley, 2010). Bacon \& Handley (2011) demonstrated that a concurrent secondary task that loads visual memory resources impaired 
reasoning accuracy for dyslexics. This was not the case for non-dyslexics, despite the groups having similar visual memory ability (as measured by the VPT) and performing similarly under low concurrent load conditions. We argued that visual resources can play a compensatory role, allowing dyslexics to perform cognitive tasks in which verbal processes would usually predominate.

However, there are inherent differences between reasoning tasks and the CBT, not only in terms of the actual task demands, but also in how they are presented in the laboratory. For tasks such as reasoning, participants are typically briefed in terms of the structure of the problem and expected conclusion, describing these in terms of premises, quantifiers etc. While this provides little clue to the most effective strategy, problems are usually presented in written form. In the absence of instructions about how information should be represented and manipulated, non-dyslexics may just use the material as it is presented. Dyslexics however may spontaneously compensate for their verbal difficulties by using a strategy which converts written information into more dyslexia-friendly visual images (e.g., Bacon et al. 2007). With the CBT, a spatial-sequential strategy is assumed to be normative and ability in this domain is what the task is designed to measure (e.g., Kessells, et al, 2008; Berch et al., 1998). This strategy is implied in describing the task to participants in terms of its presenting sequences of locations and reinforced by subsequent serial presentation of stimuli. Most participants may therefore be prompted to use this approach by default, even those completing the $\mathrm{CB}$ version first may begin practice trials using serial recall. An alternative strategy based on visual and/or executive processes may subsequently be triggered by CB task demands. Although counterbalancing should control for order effects, we checked for these statistically in view of this strategy switch explanation and found no evidence that Corsi presentation order influenced recall accuracy in either group $(p>.72)$. 
Research suggests that cognitive strategy or task switching involves the suppression of incoming information which is no longer relevant and deactivation of an established mental set in order to switch to a new one (e.g., Miyake et al., 2000; Monsell, 1996). These complex cognitive processes may present difficulty for dyslexics as they are associated with executive functions, indeed the ability to switch between tasks/strategies has been claimed as one of the most important indicators of executive control (e.g., Miyake et al., 2000; Monsell, 1996). If this proposal is correct, two possible explanations for dyslexic performance on $\mathrm{CB}$ in Experiment 1 are suggested:

- They are not able to effectively employ a strategy based on visual memory resources on this particular task, possibly because of an associated executive deficit.

- They are potentially able to use a visual strategy, but do not switch strategies and hence persevere with a spatial sequential approach throughout.

Experiments 2 and 3 aimed to test each of these possibilities. Firstly, Experiment 2 aimed to circumvent spontaneous strategy use by explicitly instructing participants to employ either a spatial-sequential or static visual pattern based strategy. If our interpretation is correct, with spatial-sequential instructions both groups should present a deficit on $\mathrm{CB}$ compared to $\mathrm{CF}$, the dyslexics because they are continuing to apply the default normative CBT strategy (as they presumably did in Experiment 1) and the non-dyslexics because they will be using a less effective CB strategy than they did in Experiment 1. Conversely, with instructions promoting a visual strategy, no significant deficit on CB should be observed - non-dyslexics should be using their default approach, namely a visually-based strategy (as suggested in Experiment 1) 
but dyslexics should also use that strategy instead of the one they would otherwise have adopted spontaneously (presumably one based on serial rehearsal). There is evidence that a visual strategy can be effective on CF too (Smyth and Scholey, 1992; Wilde et al, 2004) so these instructions should not affect this version of the task adversely. If, however, dyslexics continue to present a deficit despite visual instructions, then it will suggest that their performance in Experiment 1 may be due to an inability to actually use a strategy based on visual memory, rather than simply a failure to adopt this strategy.

\section{Experiment 2}

\section{Methods}

\section{Participants}

Seventy-six students from Plymouth University took part. They were recruited as in Experiment 1, all were native English speakers and none had taken part in Experiment 1. The participants were allocated to either dyslexic $(\mathrm{n}=38 ; 18$ males, 20 females $)$ or non-dyslexic ( $\mathrm{n}=38 ; 11$ males, 27 females) groups according to exactly the same criteria as in Experiment 1. No co-morbid conditions were disclosed in the dyslexic group and no learning/developmental disorders disclosed in the control group. The same three additional screening measures were administered and again the pattern of scores observed supported the group allocations. The two groups presented similar levels of IQ and the only differences between them were limited to those associated with dyslexia - see Appendix A.

\section{Design and Procedures}


All participants were presented with the computerised version of the standard CBT in both $\mathrm{CF}$ and $\mathrm{CB}$ conditions, as in Experiment 1. In a within-subjects design, all completed the task twice, once under each of two instruction conditions:

1. Visual: Participants were asked to actively try to remember the locations simultaneously that is, to visualise them all together as an overall pattern of squares.

2. Sequential: Participants were asked to actively try to remember locations individually as a sequence - i.e. the first square, followed by the second square, followed by the third etc.

The order of presentation of the two instruction conditions was counterbalanced, as was ordering of $\mathrm{CF}$ and $\mathrm{CB}$. After completing both $\mathrm{CF}$ and $\mathrm{CB}$ under one type of instruction, participants completed an unrelated filler task before completing the CBT again under the alternative instructions.

\section{Results}

Figure 2 illustrates that with visual instructions, both groups performed similarly on CF and $\mathrm{CB}$ while with spatial-sequential instructions they both present a noticeable reduction in recall accuracy on $\mathrm{CB}$.

\section{INSERT FIGURE 2 ABOUT HERE}

A 2 (group: dyslexic, non-dyslexic) x 2 (instruction type: visual, sequential) x 2 (recall direction: $\mathrm{CF}, \mathrm{CB}$ ) repeated measures ANOVA indicated a significant main effect of recall direction: $F(1,74)=32.02, M S E=.74, p<.001, \eta^{2}=.30$ which reflected the overall tendency to perform best on CF. A significant 2-way interaction between instruction set and 
direction was also apparent; $F(1,74)=10.86, M S E=1.11, p=.002, \eta^{2}=.13$. No other significant effects were observed $(\mathrm{p}>.3$ in every case). Post-hoc tests confirmed that with visual instructions, $\mathrm{CF}$ and $\mathrm{CB}$ scores were comparable in both groups (dyslexics $p=.47$; $\mathrm{CF} M=6.05, S D=1.11 ; \mathrm{CB} M=5.89, S D=1.31$, and non-dyslexics $\mathrm{p}=.24 ; \mathrm{CF} M=6.29$, $S D=.93 ; \mathrm{CB} M=6.11, S D=.83)$. However, with spatial-sequential instructions, both groups performed significantly less well on $\mathrm{CB}$ compared with $\mathrm{CF}$ : dyslexic $t(37)=5.06, p$ $<.001$; non-dyslexic $t(37)=2.91, p=.006$. There were no significant differences between groups in scores on either recall task in either instruction condition ( $p>.3$ in every case).

\section{Discussion}

Following Experiment 1, we hypothesised that an effective strategy for CB may be one which involves memory for static visual representations of the locations. We offered two possible explanations for dyslexics' lower performance on CB: either that they are unable to employ such a strategy, or that they can do so, but fail to switch to this approach, instead persevering with a spatial sequential strategy. The results of Experiment 2 refute the first of these suggestions. Our results show that although dyslexics may not spontaneously adopt a recall strategy based on a visual representation of spatial locations in $\mathrm{CB}$, they can do so as effectively as non-dyslexic participants if prompted. Together with the results of Experiment 1, our data support our second hypothesis, namely that individuals with dyslexia have difficulty selecting the most appropriate strategy in CB. If we assume that the default recall strategy for CBT is sequential, our hypothesis proposes that non-dyslexics are able to override this and switch to a visually-based approach in the $\mathrm{CB}$ condition. Dyslexic participants do not switch and appear to persevere with a sequential approach. 
We tested this proposal further in Experiment 3 where we manipulated the task demands to investigate whether an increased level of difficulty may force dyslexics to abandon an unsuitable sequential strategy and adopt a visual one instead. The aim was to examine whether, when faced with a task which makes their preferred strategy more difficult, dyslexics can recognise the need to change strategies and do so without the need for explicit instruction. We introduced a retention interval into the CBT, during which participants were required to carry out a task designed to interfere with the maintenance of either visual or spatial-sequential information. If people with dyslexia tend to use spatial-sequential processes and stick with them throughout, a secondary task blocking the maintenance of spatial sequences may force them to draw on compensatory processes, presumably involving static visual representations. If they are able to switch strategies effectively, then their $\mathrm{CB}$ recall should be equivalent to $\mathrm{CF}$ and comparable to that of non-dyslexic participants. Reduced recall on $\mathrm{CB}$ would suggest, on the other hand, that dyslexic participants are unable to switch strategies effectively even when the spatial sequential approach is made untenable. Nondyslexics should be relatively unaffected by the interference as long as they adopt a visuallybased strategy rather than a sequential one (given Smyth \& Scholey, 1992; Wilde et al., 2004's proposal that both CF and CB can be conducted by means of static visual representations). Conversely, a secondary task blocking the maintenance of visual information should disrupt a strategy based on remembering static visual patterns. We would expect to see non-dyslexics performance on $\mathrm{CB}$ reduced in this condition as they cannot use their preferred approach and a spatial-sequential one is likely to be less effective. As dyslexics do not appear to spontaneously adopt a visual approach anyway, in this condition they should simply present their default CB deficit. A limitation of Experiment 2 was that we were unable to check for a replication of the group differences in CBT scores observed in 
Experiment 1. Therefore, in Experiment 3, we also presented a standard (no interference) version of the CBT and the VPT.

\section{Experiment 3}

\section{Methods}

\section{Participants}

Seventy-one students from Plymouth University took part. They were recruited by the same means as previously and again all were native English speakers. None had taken part in Experiments 1 or 2. Exactly the same criteria were used to assign participants to either the dyslexic ( $n=34,15$ males, 19 females) or non-dyslexic ( $n=37 ; 11$ males, 26 females) groups. No co-morbid conditions were disclosed in the dyslexic group and no learning/developmental disorders were disclosed in the control group. The same three additional screening measures were administered and results of these suggest our sampling was valid, see Appendix A.

\section{Design and Procedures}

Participants were first presented with a computerised version of the standard Corsi task as described in Experiment 1. After a short break, $\mathrm{CF}$ and $\mathrm{CB}$ were administered again. However, in this case, during each trial a 10s retention interval was introduced after presentation of the to-be-remembered sequence, and before the participant was asked to recall it. During the retention interval participants performed a task designed to interfere with maintenance of either spatial-sequential or visual information. All participants performed both $\mathrm{CF}$ and $\mathrm{CB}$ with visual and spatial interference, the order of presentation counterbalanced across participants. The interference tasks were selected as they have 
previously been shown to selectively disrupt processing on static-visual and dynamic spatialsequential primary tasks respectively in investigations of visual vs. spatial memory using the VPT and CBT (Della Sala et al., 1999):

The Visual interference task required participants to view irrelevant pictures during the retention interval of the Corsi tasks. Twelve A4 size cards were prepared, each containing a print of a painting taken from the work of Wassily Kandinsky, an avant-garde artist noted for his colourful, abstract work. The paintings were chosen because they are difficult to encode verbally and dissimilar to the patterns used in the VPT or the visual pattern participants may remember in the Corsi task. Each participant viewed one painting card per trial, with paintings presented in a different random order for each participant. This type of visual task has been widely used in a similar context (e.g. Logie, 1986; Logie \& Pearson, 1997). Della Sala et al (1999) showed that it selectively interferes with recall on the VPT but not on the CF task, suggesting that it successfully taps into visual, rather than spatial, memory resources.

The Spatial interference task required participants to follow, by touch alone, an arrangement of small wooden pegs. The apparatus consisted of $10 \mathrm{x} 10 \mathrm{~cm}$ wooden board on which were set 5 cylindrical pegs, each $6 \mathrm{~cm}$ tall and $1.5 \mathrm{~cm}$ in diameter. The pegs were positioned one to each corner of the board and one in the centre. The board was placed behind a screen with a gap beneath, which allowed participants to handle the board without being able to see it. The task was to follow haptically the sequence of pegs in a figure-of-eight pattern. This is a wellestablished dynamic spatial task, based on a technique developed by Smythe and colleagues (e.g., 1988; 1989). Della Sala et al (1999) showed that when used as a secondary measure, this task interferes with recall on CF, but not on the VPT, suggesting that it is successful at tapping into dynamic-spatial, as opposed to static-visual, short-term memory resources. 
Finally, all participants completed the VPT as described in Experiment 1.

\section{Results}

Figure 3 presents recall performance on the standard (no interference) CBT and across the two interference conditions.

\section{INSERT FIGURE 3 ABOUT HERE}

Data on the standard task presented a clear replication of Experiment 1. A 2 group (dyslexic; non-dyslexic) x 2 recall direction $(\mathrm{CF}$; $\mathrm{CB})$ ANOVA presented significant main effects of recall direction, $F(1,69)=23.39, M S E=.69, p<.001, \eta^{2}=.29$ and of group, $F(1,69)=$ 5.86, MSE $=.93, p<.001, \eta^{2}=.08$, together with an interaction between these factors, $F(1$, 69) $=14.81, M S E=.69 . p<.001 . \eta^{2}=.18$. Simple within-groups comparisons confirmed that non-dyslexics performed similarly on $\mathrm{CF}(M=6.32, S D=.94)$ and $\mathrm{CB}(M=6.12, S D=$ $1.00 ; p>.4)$. The dyslexic group, however, recalled significantly fewer locations on CB (M $=5.19, \mathrm{SD}=.66)$ compared to $\mathrm{CF}(\mathrm{M}=6.47, \mathrm{SD}=.93 ; \mathrm{p}<.001)$. Between-group comparisons confirmed comparable scores on $\mathrm{CF}(\mathrm{p}>.51)$ while non-dyslexics performed significantly better than dyslexics on CB: $\mathrm{t}(69)=4.55, \mathrm{p}<.001$. Also in line with Experiment 1, the two groups performed comparably on the VPT (dyslexics $M=10.29, S D=$ 2.05; non-dyslexics $M=10.87, S D=1.82 ; t(69)=1.27, p>.2$ ). Table 1 shows that VPT score correlated significantly with $\mathrm{CB}$ recall for non-dyslexics only and the between-group difference in correlations was highly significant. There was little evidence of a relationship between VPT and CF scores for either group. 
As Figure 3 shows, in the visual interference condition, both groups performed less well on $\mathrm{CB}$ compared to $\mathrm{CF}$ (non-dyslexics, $\mathrm{CF} M=5.22, S D=1.53$ and $\mathrm{CB} M=4.35, S D=1.08$; dyslexics $(\mathrm{CF} M=4.94, S D=1.41$ and $\mathrm{CB} M=4.26, S D=1.08)$. However, with spatialsequential interference, this effect was only observed for dyslexics (CF $M=4.68, S D=1.47$; CB $M=3.44, S D=1.52)$, non-dyslexics performed similarly in both recall directions (CF $M$ $=4.30, S D=1.45 ; \mathrm{CB} M=4.16, S D=1.48$ ). A 2 (group: dyslexic, non-dyslexic) $\mathrm{x} 2$ (interference: visual, spatial) x 2 (recall direction: $\mathrm{CF}, \mathrm{CB}$ ) repeated measures ANOVA indicated a significant main effect of interference type: $F(1,69)=11.93, M S E=1.79, p=$ $.001, \eta^{2}=.15$, reflecting the overall better performance observed in the visual interference condition compared to the spatial interference condition. A main effect of recall direction; $F$ $(1,69)=40.69, M S E=.93, p<.001, \eta^{2}=.37$, illustrated an overall trend to perform better on forwards recall. A significant interaction between dyslexic status and recall direction was observed; $F(1,69)=3.99, M S E=.92, p=.05, \eta^{2}=.06$, as well as a significant 3-way interaction; $F(1,69)=10.48, M S E=.70, p=.002, \eta^{2}=.13$. These effects were further examined in some within-group comparisons. In the non-dyslexic data, significant main effects of interference type: $F(1,36)=7.45, M S E=1.53, p=.01, \eta^{2}=.17$, and recall direction, $F(1,36)=13.88, M S E=.67, p=.001, \eta^{2}=.28$ were indicated. A significant interaction between these factors was also observed, $F(1,36)=6.74, \operatorname{MSE}=.73, p=.01, \eta^{2}$ $=.16$, illustrating that the pattern of recall performance on $\mathrm{CF}$ and $\mathrm{CB}$ differed significantly across interference conditions for the non-dyslexics. In the dyslexic group, the main effects were again observed, interference type, $F(1,33)=4.84, M S E=2.08, p=.03, \eta^{2}=.13$ and recall direction, $F(1,33)=25.83, M S E=1.20, p<.001, \eta^{2}=.44$. In this case however, the interaction was not significant, $F(1,33)=3.96, M S E=.67, p=.08, \eta^{2}=.11$, so, in contrast to the non-dyslexics, dyslexic participants present no significant difference in their pattern of 
recall across the two interference conditions. As Figure 3 illustrates, in the visual interference condition non-dyslexics performed significantly better on CF than CB: $t(36)=4.64, p<.001$ while with spatial-sequential interference their recall across the two tasks was comparable ( $p$ $=.51)$. The dyslexics on the other hand, recalled fewer items on $\mathrm{CB}$ compared to $\mathrm{CF}$ in both conditions, with visual interference: $t(33)=4.94, p<.001$, and with spatial-sequential interference: $t(33)=3.1, p=.004$. A single ANOVA incorporating all three interference conditions produced results consistent with those above ${ }^{1}$.

Finally, Table 1 presents the results of correlational analyses which demonstrate the relationship between VPT and CBT span under the two interference conditions. Dyslexics show no significant associations at all, while non-dyslexics present significant correlations between VPT and both CF and CB span with spatial interference. The between-group difference in correlation coefficients in this condition was significant for $\mathrm{CB}$, though not for CF.

\section{Discussion}

Following Experiments 1 and 2, we suggested that non-dyslexics switch to a visual strategy on $\mathrm{CB}$ and so preserve their recall performance. As such, preventing the switch by blocking a visual strategy should result in reduced CB recall for these participants. Our results supported this hypothesis. Conversely, we predicted that this $\mathrm{CB}$ deficit would not be observed with spatial-sequential interference because both recall tasks $(\mathrm{CF}$ and $\mathrm{CB})$ can theoretically be conducted using a visual approach (Smyth \& Scholey, 1992; Wilde et al, 2004). Accordingly, we found non-dyslexic recall across the two tasks to be comparable in this condition. For dyslexics, on the other hand, our suggestion following Experiments 1 and 2 was that they fail 
to make the switch from a spatial-sequential to a visual strategy for $\mathrm{CB}$, and their $\mathrm{CB}$ recall was reduced as a result. In Experiment 3 we therefore predicted that visual interference would make little difference to their approach - they would simply use a spatial-sequential strategy throughout and hence recall fewer items correctly on $\mathrm{CB}$ compared to $\mathrm{CF}$, just as they had been found to do in the previous studies - the data supported this prediction. Conversely, we expected that spatial-sequential interference would hamper the dyslexics' natural strategy. If they were able to switch to a visual strategy, these increased demands would force them to do so and, as the visual approach is effective for both $\mathrm{CF}$ and $\mathrm{CB}$, their recall would be comparable. However, our data suggested otherwise as (in contrast to nondyslexics) dyslexic participants again performed significantly less well on CB compared to CF. It appears that even when prevented from applying a spatial-sequential strategy, dyslexic participants were unable to switch effectively to an alternative approach for $\mathrm{CB}$ and, as a result, their performance was impaired.

These proposals are supported by data in the standard no-interference CBT condition. Importantly, we were able to replicate the results of Experiment 1, in terms of both the overall pattern of recall accuracy and the relationships between this and VPT score. These data suggest a role for visual short-term memory in $\mathrm{CB}$ recall for non-dyslexics, those with higher ability in this domain performing to level similar to that observed in CF. This pattern of recall accuracy is also observed with spatial-sequential interference, where a relationship between VPT score and both $\mathrm{CF}$ and $\mathrm{CB}$ is also evident, further suggesting that these participants are able to draw on visual memory resource when spatial resource is constrained. No such relationships are observed for dyslexics and they are less accurate on CB compared to $\mathrm{CF}$ throughout. While these correlational data cannot be considered definitive evidence for visual vs. spatial recall strategies, when considered alongside CBT recall accuracy they do 
offer some substantiation for the proposal that non-dyslexics routinely use visual processes for $\mathrm{CB}$, and for $\mathrm{CF}$ where necessary, while dyslexics use a more spatial recall strategy which breaks down under high spatial demand conditions. Moreover, the correlations also suggest that our interference tasks were effective (there is no evidence of a relationship between visual memory ability and CBT recall for either group with visual interference).

\section{General Discussion}

We carried out three experiments comparing the performance of individuals with and without dyslexia on variations of the Corsi blocks visuospatial memory task. It is often claimed that dyslexia is characterised by verbal serial memory deficits but intact, or even enhanced, visuospatial memory performance. Our Experiment 1 suggested that both groups do indeed perform comparably on both $\mathrm{CF}$ and the Visual Patterns Test, measuring serial visuospatial and visual short-term memory respectively. However, a different picture emerged in the backward version of the Corsi task where dyslexic participants performed significantly worse than non-dyslexics. The correlations suggested a positive relationship between visual memory span and $\mathrm{CB}$ recall for the non-dyslexics and we hypothesised that when faced with the processing demands of backward recall, this group may tend to draw on their visual alibies, abandoning a serially-based spatial strategy in favour of a more efficient visuallybased one. Conversely, we attribute the poorer performance of dyslexic participants on $\mathrm{CB}$ to a failure to adapt to task demands. Equivalent VPT scores across the two groups of participants provided further evidence that the dyslexics low performance on $\mathrm{CB}$ is likely due to a failure to adopt a visual strategy, rather than a deficit in visual memory per se. Experiment 2 supported this suggestion by demonstrating that dyslexic participants perform as non-dyslexics on $\mathrm{CB}$ if explicitly instructed to adopt a visual strategy. Conversely, non- 
dyslexic participants exhibited a deficit on $\mathrm{CB}$ if instructed to perform the task using a spatial-sequential strategy. Finally, Experiment 3 provided further converging evidence to support these results. In this study, control data was available which presented a clear replication of Experiment 1. The strategic distinctions implied by this are supported by the experimental data which showed that dyslexic participants still fail to adopt a visual strategy in $\mathrm{CB}$ even with the imposition of a secondary task which inhibits spatial-sequential processing.

Several previous studies have suggested that dyslexic participants exhibit difficulties in tasks which place demands on executive functions (Reiter, et al., 2004; Smith-Spark \& Fisk, 2007; Swanson, et al., 2009). Executive functions are thought to play a role in CB (e.g., Vandierendonck, et al., 2004) and our findings are therefore consistent with an executive deficit in dyslexic participants. Our research goes further, however, by demonstrating that this executive function deficit takes the form of a failure to adopt a visual strategy in CB. When explicitly instructed to do this, dyslexic and non-dyslexic participants perform equally well. Although dyslexics have been found to spontaneously adopt strategies based on visual processes in other domains such as reasoning (e.g., Bacon et al., 2007; 2010a; b), we suggest that the problem in $\mathrm{CB}$ may arise because the Corsi task may naturally prompt participants to use a sequential recall strategy due to the sequential nature of the stimuli presentation. As such, dyslexics may experience difficulty in the process of switching from a default serial strategy (which they use as efficiently as non-dyslexic participants in CF) to a more effective visual approach. We suggest that it is in this strategy switching that the executive deficits become manifest. 
Miyake et al. (2000) described how task or strategy switching may involve three distinct executive processes: updating, inhibition and shifting. There is evidence that these functions are impaired in dyslexics. Updating requires the monitoring of incoming information for its relevance to the task at hand and then encoding this new information through the continuous revision of the content of working memory. In this way, old, no longer relevant, information is replaced by that which is newer and more relevant. This requires an active manipulation of information in working memory, rather than simply passive, short-term storage. Smith-Spark and colleagues (2007) presented sequences containing varying numbers of stimuli and participants were asked to recall the $x$ most recent items. Successful task performance required the participants to hold the first $x$ items in memory and then, if there were more than $x$ items in a list, to update the contents of memory by dropping the least recent item and adding the new item to the string. Smith-Spark et al. presented evidence of a deficit in this task in adults with dyslexia. Similarly, deficits in the ability to deliberately inhibit dominant, automatic, or prepotent responses may also be impaired in dyslexia. Inhibition is often tested by a Stroop task, in which participants are required to override the tendency to name a colour word printed in a non-complementary colour. Dyslexics are known to present Stroop deficits, with dyslexic children presenting larger levels of interference than age matched nondyslexics (e.g., Everatt, Warner, Miles \& Thomson, 1997; Protopapas, Archonti \& Skaloumbakas, 2007).

Finally, Miyake et al discuss a shifting process which requires the disengagement of an irrelevant task set and the subsequent active engagement of a new, more relevant one. Individual differences may not be a simple reflection of the ability to engage and disengage task sets per se, but may also (or even instead) involve the ability to perform a new operation in the face of proactive interference or negative priming. In terms of our results, with the 
standard CBT, as administered in Experiments 1 and 3, task demands may inadvertently cue a sequential strategy which subsequently requires a strategy shift for $\mathrm{CB}$, facilitated by the $\mathrm{CE}$. The possibility of a shifting deficit is supported by the results of Experiment 2, which showed that participants with dyslexia were capable of using an alternative visual strategy in $\mathrm{CB}$ when explicitly instructed to do so. In that case, dyslexic participants performed as well as non-dyslexics. Presumably because the instruction circumvented the initiation of a sequential approach at the start of the Corsi task, shifting was not required and dyslexics were able to use visual processes to effect. Task switching, specifically the relationship between shifting ability and CBT performance, may prove a fruitful area for future research. Another possibility for future research might be to collect verbal accounts to describe how participants approached the task. Protocol data describing the qualitative nature of thinking in dyslexia has proved informative in previous research (e.g. Bacon et al, 2007) and in the CBT this might elucidate the nature of strategies employed under selective interference conditions and how dyslexics attempt to manage the shifting process.

Swanson (1989) has shown that although people with reading disabilities such as dyslexia often have the cognitive skills and abilities to perform a given task, they are unable to draw on them in a way which allows for the development of an appropriate strategy. Swanson suggested that such individuals do not possess the cognitive flexibility to organise and plan effective use of cognitive resources in the same way as their non-dyslexic peers. In our study, this may be reflected in an inability to organise cognitive resources in order to draw on intact visual abilities. Furthermore, Meltzer (1991) suggested that dyslexics may lack the cognitive flexibility to be able to access metacognitive information efficiently. As such, they may not possess the metacognitive awareness to even recognise the usefulness of certain strategies for a given task. In our Experiment 3, even when spatial-sequential processes were made 
unworkable by the task demands, participants with dyslexia were still unable to switch strategies. Strategy generation, selection and flexibility are all processes associated with executive functions. A recent meta-analysis (Johnson et al., 2010) reviewed studies on cognitive processing in students with learning disabilities (including those associated with dyslexia) and concluded that the evidence for cognitive processing and strategic differences is sufficient to warrant testing as part of the formal assessment process.

Our studies therefore raise some interesting questions about the potential for strategy instruction to improve recall. Several studies have investigated the effect of implicit cues to recall and have found that, when provided with cues, students with dyslexia can increase recall performance levels to those of non-dyslexics (e.g., Swanson et al., 1991; Torgesen \& Goldman, 1977; Wong, 1978). Much of this research presented verbal information, an exception being Swanson (1984) who found that students with dyslexia performed better than a control group when cued to retrieve complex visual information that could not be encoded verbally. However, cues frequently target the recall of a particular item or location within a sequence, rather than a recall strategy and future research investigating the latter would be welcome. Given that much extant research suggests that dyslexics are often inherently visual thinkers, the possibility of presenting a form of visual cue to stimulate the involvement of visual resources in subsequent recall of information may be of potential interest.

Successfully cuing a recall strategy may have implications beyond formal cognitive testing. People with dyslexia are known to experience difficulties with memory during many ordinary everyday activities, such as recalling a range of naturalistic objects (Swanson, et al. 1991) remembering details of everyday events, social situations, and procedures such as checking out a book from the library (McNamara and Wong, 2003). These results are often explained 
in terms of an executive deficit. Interestingly in terms of our present discussion, when McNamara and Wong compared their participants on cued recall, the between group difference was significantly reduced. Like our present results, this indicated that, given some kind of prompt, people with dyslexia can recall information as well as non-dyslexics. McNamara and Wong suggest that it is the search and retrieval strategies which seem to be inefficient - in other words, the ways in which people with dyslexia attempt to locate and recall material in long term memory, again likely to be a strategic process under executive control.

However, in everyday life, although dyslexics are known to experience more memory lapses than non-dyslexics (McNamara \& Wong, 2003; Smith-Spark et al., 2004) there is anecdotal evidence that they can and do draw spontaneously on visual strategies to assist them. For instance, Davis (1997), West (1997) and Morgan and Klein (2000) have written extensively about individuals for whom visualisation is a primary mode of thought, compensating for memory difficulties in the verbal domain when remembering written or auditorially presented information (see also Grant, 2005). The first author has worked with numerous adults with dyslexia who report remembering numbers (such as cashpoint identification numbers or phone numbers) not as strings of digits but by visualising the pattern they make according to their position on a standard keypad. This illustrates that in everyday situations, many dyslexics do possess sufficient metacognitive awareness to create effective compensatory recall strategies, and that these strategies are inherently visual in nature.

Our results are also of interest in terms of theoretical and methodological issues in understanding visuospatial WM. We provide support for previous research which has suggested a role for other than spatial sequential processes in the CBT (e.g., Vandierendonck 
et al., 2004; Mammarella \& Cornoldi, 2005). From the WM model perspective, CF assesses the ability to maintain visuospatial information for a brief period of time and is, as such, a good measure of general VSSP capacity. However, CB requires both maintenance and manipulation of order information, which relies not only on the VSSP but also the central executive. It is likely that this is not simply a matter of drawing on additional resources for longer or more difficult sequences, but may implicate an overall strategy switch and our data suggest that a strategy based on a static visual pattern of locations is adaptive for CB. Some recent research by Ang and Lee (2008) with normally developing children has demonstrated CE involvement in a range of spatial memory measures, including the CBT and these results are explained in terms of interdependence between spatial short-term memory and executive functioning. This idea is interesting with regard to our present discussion for two reasons. Firstly, Ang and Lee only presented the CF recall condition, suggesting that CE involvement may not be limited to $\mathrm{CB}$. This raises further questions about processes involved in $\mathrm{CF}$ because, despite their frequently documented executive impairments, dyslexics generally perform well on this task. Secondly, Ang and Lee selected their measures for their specifically spatial (rather than visual) nature, based on the premise of a fractionated VSSP. They do not examine the visual subcomponent at all and similar research to investigate this domain, in both dyslexic and neurotypical populations, would be worthwhile, particularly in view of the idea that the $\mathrm{CE}$ itself may be fractionable into distinct visual and spatial systems (e.g., Miyake et al, 2001). A relatively recent development of the WM model has been the incorporation of the episodic buffer, a limited capacity temporary storage facility that integrates long-term memory with the working memory slave systems, thus freeing up CE capacity (Baddeley, 2000). The buffer is thought to be controlled by the CE, but the extent to which it is intact in dyslexia, and the role it plays in working memory for such individuals, remains unclear. 
Additionally, there are potential implications for administration of the CBT. We suggest the possibility that task demands may inadvertently cue a sequential strategy. This may become an issue in versions of CBT which require different or additional processes (such as CB) and with participants who are less able to switch strategies. In this case, the CB score may not reflect the participant's memory capacity per se but, at least in part, his/her strategic flexibility. Given that individuals with dyslexia are consistently found to present executive deficits, to claim that a lack of deficit on CF represents an intact visuospatial memory resource, whilst not also administering $\mathrm{CB}$, neglects a potentially rich additional source of data. Kessels et al. (2008) have highlighted how, because the CBT was designed as an analogue of the verbal digit span, it is appropriate to include both forward and backward span procedures in order to make the two paradigms comparable - although, as we have seen, recall on CBT may reflect far more than capacity within a unitary VSSP. Furthermore, while our results support Della Sala et al (1999) in highlighting that CF and VPT likely draw on distinct spatial and visual resources respectively, we additionally suggest that both VPT and CB performance might reflect capacity in the visual subcomponent of the VSSP, with CB drawing on additional CE resource when required.

However, there are some possible limitations of our studies. Firstly, we had no control data in Experiment 2 which meant that we could not be certain that the group differences in standard CBT performance observed in Experiment 1, and hence the recall strategies inferred from them, were replicated. However, the results of the first Experiment informed predictions for the second, and these predictions were upheld in our instruction conditions which suggest that our á priori assumptions are likely correct. Furthermore, In Experiment 3, control data is available which reflects clear replication of Experiment 1 and the experimental data again 
supports our thesis. Overall, taken together, the three studies present consistent evidence that strategy related deficits underpin dyslexic performance on CB. A second potential limitation is that we didn't control statistically for the presence of attention deficit disorder (ADD). ADD is known to co-occur with language and learning disabilities (including dyslexia), with co-morbidity rates of between 25 and 40\% cited (e.g. Dykman \&Ackerman, 1991; Willcutt \& Pennington, 2000). This is a potential concern because individuals with ADD have been found to exhibit impairment in verbal and spatial storage and deficits in the visual subcomponent of CE (Seidman, Biederman, Weber, Hatch \& Faraone, 1998) even with comorbid language deficits controlled for (Martinussen and Tannock, 2006). However, none of our participants declared a diagnosis of ADD when asked, and there is no reason to suppose that non-declaration is any less accurate than has been shown to be the case for dyslexia (Nicolson and Fawcett, 1997). Nonetheless, any undisclosed ADD among dyslexic participants may have influenced our findings. Their inability to draw on visual memory processes in $\mathrm{CB}$ might be attributable to attention related difficulties with visual storage (for instance, maintaining a visual pattern trace) or in visual components of the $\mathrm{CE}$ required for task switching. Future work might usefully control more formally for co-morbid ADD, for instance by administering a measure such as the Adult ADHD Self-Report scale (AASRS; Kessler, et al, 2005) or the Brown ADD scale for child participants (Brown, 2001).

Finally, our data cannot account for the possibility that some participants may have used verbal coding under high task demands. This strategy has been observed in children and Pickering et al (2001) discuss how it may be linked to distinct developmental trajectories of verbal and visuospatial memory. In adult participants the evidence is equivocal. Vandierendonck et al (2004) report evidence for verbal coding in CF but were unable to replicate the effect in a later study, while Kessels et al (2008) argued that verbal and 
visuospatial working memory could be clearly dissociated, with digit span and CBT measuring distinct cognitive processes. Given that dyslexics present significant verbalphonological deficits and that they are known to favour visual strategies in domains where the more normative approach is verbal (Bacon et al, 2007; 2010a) it would seem unlikely that they routinely attempt a verbal coding of CBT stimuli. However, further research might usefully explore effective verbal coding of visuospatial stimuli as a possible index of difference between dyslexic and non-dyslexic populations.

In conclusion, our results support the idea of a central executive deficit in dyslexia, rather than a specific impairment in the visual or spatial subcomponent of short-term memory. This executive deficit is manifested as a difficulty in adopting the most appropriate strategy for the recall task (a visually-based strategy). The results of our research suggest that if induced by instructions to draw on an effective strategy, not only are dyslexics able to do so, but their recall performance can be improved to reach the level observed in non-dyslexic participants. In educational settings, where the recall of information plays a key role in academic success, Deschler, Ellis and Lenz (1996) described how interventions to support students with learning difficulties can include components to help compensate for memory-related difficulties, improve metacognitive awareness and strategic flexibility. Our results support the drive for teachers and lecturers to develop a repertoire of teaching and learning strategies which enable individuals to draw on intact cognitive resources and abilities, and to generate and employ cognitive strategies most appropriate to the tasks at hand. 


\section{References}

Aaron, P. G. \& Guillemond, J. C. (1993). Artists as dyslexics. In D.M. Willows, R.S. Kruk \& E. Corcos (Eds.). Visual Processes in Reading \& Reading Disabilities (pp. 393-415). Hillsdale, NJ: Erlbaum.

Ackerman, P.T. \& Dykman, R.A. (1993). Phonological Processes, confrontational naming and immediate memory in dyslexics. Journal of Learning Disabilities, 26(9), 567-609. and skilled readers. Journal of Abnormal Child Psychology, 19(2), 117-147.

Ang, S.Y. \& Lee, K. (2008). Central executive involvement in children's spatial memory. Memory, 16(8), 918-933.

Bacon, A.M. \& Handley, S.J. (2010a). Dyslexia and reasoning: The importance of visual processes. British Journal of Psychology, 101, 433-452.

Bacon, A.M. \& Handley, S.J. (2010b). Dyslexia, reasoning \& visual-spatial processes. In N. Alexander-Passe (Ed). Dyslexia and Creativity: Investigations from Differing Perspectives (pp. 25-49). Nova Science.

Bacon, AM., Handley, S.J. \& McDonald, E.L. (2007). Reasoning and dyslexia: a spatial strategy may impede reasoning with visually rich information. British Journal of Psychology. 98(1), 79-92. 
Baddeley, A. D. (2000). The episodic buffer: A new component of working memory? Trends in Cognitive Sciences, 4(11), 417 - 423.

Baddeley, A.D. (2007). Working Memory, Thought \& Action. Oxford: Oxford University Press.

Baddeley, A.D. and Hitch, G.J. (1974) Working memory. In G.A. Bower (Ed.). The Psychology of Learning and Motivation (pp. 47-89). ,Academic Press.

Baddeley, A.D. \& Logie, R.H. (1999). Working memory: the multiple component model. In A. Miyake \& P. Shah (Eds.). Models of working memory: mechanisms of active maintenance and executive control (pp. 28-60). Cambridge: Cambridge University Press.

Bayliss, D. M., Jarrold, C., Gunn, M. D., \& Baddeley, A. D. (2003). The complexities of complex span: Explaining individual differences in working memory in children and adults. Journal of Experimental Psychology: General, 132, 71-92.

Beneventi, H., Tønnessen, F,E., Ersland, L. \& Hugdahl, K. (2010). Working Memory Deficit in Dyslexia: Behavioral and fMRI Evidence. International Journal of Neuroscience, $120(1), 51-59$.

Berch, D.B., Krikorian, R. \& Huha, E.M. (1998). The corsi block-tapping task: methodological and theoretical considerations. Brain and Cognition, 38, 317-338. 
Brosnan, M., Demetre, J., Hamill, S., Robson, K., Shepherd, H., \& Cody, G. (2002). Executive functioning in adults and children with developmental dyslexia. Neuropsychologia, 40, 2144_2155.

Brown, T.E. (2001), The Brown Attention Deficit Disorder Scales. San Antonio, TX: Psychological Corporation.

Carlesimo, G.A., Perri, R., Turriziani, P. Tomaiuolo, F. \& Caltagirone, C. (2001). Remembering what but not where: independence of spatial and visual working memory in the human brain. Cortex, 37, 519-537.

Corsi, P.M. (1972). Human memory and the medial temporal region of the brain. Dissertation Abstracts International, 34, 819B.

Courtney, S.M., Ungerleider, L.G., Keil, K. \& Haxby, J.V. (1996). Object and spatial visual working memory activates separate neural systems in human cortex. Cerebral Cortex, 6, $39-49$.

Davis, R. D. (1997). The Gift of Dyslexia. London: Souvenir Press.

Della Sala, S., Gray, C., Baddeley, A., Allamano, N. \& Wilson, L. (1999). Pattern span: a tool for unwelding visuospatial memory. Neuropsychologia, 37, 1189-1199.

Della Sala S., Gray C, Baddeley A, Wilson L. (1997). The Visual Patterns Test: A new test of short-term visual recall. London: Thames Valley Test Company. 
Démonet, J-F., Taylor, M. J., \& Chaix, Y. (2004). Developmental dyslexia. Lancet, 363, 1451 $-1460$

Deschler, D. D., Ellis, E. S., \& Lenz, B. K. (1996). Teaching adolescents with learning disabilities: Strategies and methods. Parsippany, NJ: Good Apple.

Dykman, R.A. \& Ackerman, P.T. (1991). Attention deficit disorder and specific reading disability: Separate but often overlapping disorders. Journal of Learning Disabilities, 24, 96-103.

Engle, R. W., Cantor, J., \& Carullo, J. J. (1992). Individual differences in working memory and comprehension: A test of four hypotheses. Journal of Experimental Psychology: Learning, Memory and Cognition, 18, 972-992.

Everatt, J., Warner, J., Miles, T.R. \& Thomson, M.E. (1997). The Incidence of Stroop Interference in Dyslexia. Dyslexia, 3, 222-228.

Fawcett, A. J., \& Nicolson, R. I. (1998). The Dyslexia Adult Screening Test (DAST). London: The Psychological Corporation.

Galaburda, M. S. (1993). Dyslexia and development: Neurobiological Aspects of Extraordinary Brains. Cambridge, MA: Harvard University Press.

Gould, J.H. \& Glencross, D.J. (1990). Do children with a specific reading disability have a general serial-ordering deficit? Neuropsychologia, 28(3), 271-278. 
Grant, D. (2005). That's the Way I Think: Dyslexia, Dyspraxia \& ADHD Explained (2 ${ }^{\text {nd }}$. ed.). London: Routledge.

Jeffries, S. \& Everatt, J. (2004). Working memory: its role in dyslexia and other specific learning difficulties. Dyslexia, 10, 196-214.

Johnson, E. S., Humphrey, M., Mellard, D.F., Woods, K. \& Swanson, H. L. (2010). Cognitive Processing Deficits \& Students with Specific Learning Disabilities: A Selective Meta-Analysis of the Literature. Learning Disability Quarterly, 33(1), 3-18.

Kemps, E. (1999). Effects of complexity on visuospatial working memory. European Journal of Cognitive Psychology, 11, 335-356.

Kemps, E. (2001). Complexity effects in visuospatial working memory: Implications for the role of long-term memory. Memory, 9(1), 13-27.

Kessels, R.P., van den Berg, E., Ruis, C. \& Brands, A. M. (2008). The Backward Span of the Corsi Block-Tapping Task and Its Association With the WAIS-III Digit Span. Assessment, $15(4), 426-434$.

Kibby, M.Y., Marks, W., Morgan, S. \& Long, C.J. (2004). Specific impairment in developmental reading disabilities: a working memory approach. Journal of Learning Disabilities, 37, 349-363. 
Lefly, D.L. \& Pennington, B.F. (1991). Spelling errors and reading fluency in compensated adult dyslexics. Annals of Dyslexia, 41, 143-163.

Logie, R.H. (1986). Visuo-spa tial processing in working memory. Quarterly Journal of Experimental Psychology, 38A, 229-247.

Logie, R.H. (1995). Visuo-spatial Working Memory. Hove: Lawrence Erlbaum Associates.

Logie, R.H. \& Pearson, D.G. (1997). The inner eye and the inner scribe of visuospatial working memory: evidence from developmental fractionation. European Journal of Cognitive Psychology, 9, 241-257.

Mammarella, I.C. \& Cornoldi, C. (2005). Sequence and space: the critical role of a backward spatial span in the working memory deficit of visuospatial learning disabled children. Cognitive Neuropsychology, 22, 1055-1068.

Martinez Perez, T., Majerus, S., Mahot, A. \& Poncelet, M. (2012). Evidence for a Specific Impairment of Serial Order Short-term Memory in Dyslexic Children. Dyslexia, DOI: 10.1002/dys. 1438 .

Martinussen, R. \& Tannock, R. (2006): Working memory ompairments in children with Attention-Deficit Hyperactivity Disorder with and without comorbid language learning Disorders. Journal of Clinical and Experimental Neuropsychology, 28(7), 1073-1094. 
McLoughlin, D., Fitzgibbon, G. \& Young, V. (1994). Adult Dyslexia: Assessment, Counselling and Training. London: Whurr.

McNamara, J.K. \& Wong, B. (2003). Memory for Everyday Information in Students with Learning Disabilities. Journal of Learning Disabilities, 36(5), 394-406.

Meltzer, L. (1991). Problem-solving strategies and academic performance in learning disabled students: do subtypes exist? In L.V. Feagans, E.J. Short \& L.J. Meltzer (Eds.). Subtypes of Learning Disabilities: Theoretical Perspectives and Research (pp. 163-188). Hillsdale, MJ: Erlbaum.

Miles, T. R. (1993). Dyslexia: The Pattern of Difficulties (2 ${ }^{\text {nd }}$. ed.) London: Whurr.

Miller-Shaul, S. (2005). The characteristics of young and adult dyslexic readers on reading and reading related cognitive tasks as compared to normal readers. Dyslexia, 11, 132-151.

Miyake, A., Friedman, N. P., Emerson, M.J., Witzki, A.H. , Howerter, A. \& Wager, T.D. (2000). The Unity and Diversity of Executive Functions and Their Contributions to Complex “'Frontal Lobe”' Tasks: A Latent Variable Analysis. Cognitive Psychology 41, 49-100.

Miyake, A., Friedman, N. P., Rettinger, D., Shah, P., \& Hegerty, M. (2001). How are visuospatial working memory, executive functioning, and spatial abilities related? A latentvariable analysis. Journal of Experimental Psychology: General, 130(4), 621-640. 
Monsell, S. (1996). Control of mental processes. In V. Bruce (Ed.), Unsolved mysteries of the mind: Tutorial essays in cognition (pp. 93-148). Hove, UK: Erlbaum.

Morgan, E., \& Klein, C. (2000). The Dyslexic Adult in a Non- dyslexic World. London: Whurr.

Mortimore, T. \& Crozier, R. (2006). Dyslexia and difficulties with study skills in higher education. Studies in Higher Education, 31(2), 235-251.

Nicolson, R.I. \& Fawcett, A.J. (1997). Development of objective procedures for screening and assessment of dyslexic students in higher education. Journal of Research in Reading, 20(1), 77-83.

Nicolson, R.I. \& Fawcett, A.J. (2001). Dyslexia as a learning disability. In Fawcett, A. (Ed). Dyslexia: Theory and Good Practice (pp. 141-159). London: Whurr.

Palmer, S. E. (2000). Phonological recoding deficit in working memory of dyslexic teenagers. Journal of Research in Reading, 23(1), 28-40.

Parmentier, F. B. R. (2011). Exploring the determinants of memory for spatial sequences. In A. Vandierendonck \& A. Smaleck (Eds.), Spatial Working Memory (pp. ). Psychology Press: Hove. 
Parmentier, F. B. R., \& Andrés, P. (2006). The impact of path crossing on visuospatial serial memory: Encoding or rehearsal effect? Quarterly Journal of Experimental Psychology, 59, $1867-1874$.

Parmentier, F. B. R., Andrés, P., Elford, G., \& Jones, D. M. (2006). Organisation in visuospatial serial memory: Interaction of temporal order with spatial and temporal grouping. Psychological Research, 70, 200-217.

Parmentier, F. B. R., Elford, G., \& Maybery, M. T. (2005). Transitional information in spatial serial memory: Path characteristics affect recall performance. Journal of Experimental Psychology: Learning, Memory, \& Cognition, 31, 412-427.

Pickering, S.J. (2001). Cognitive approaches to the fractionation of visuospatial working memory. Cortex, 37, 457-473.

Pickering, S. (2006). Working memory in dyslexia. In T. Alloway, \& S. Gathercole (Eds.), Working Memory and Neurodevelopmental Disorders (pp. 7-40). New York, NY: Psychology Press.

Pickering, S. J., Gathercole, S. E., Hall, M., \& Lloyd, S. A. (2001). Development of memory for pattern and path: Further evidence for the fractionation of visual and spatial short-term memory. Quarterly Journal of Experimental Psychology: Human Experimental Psychology, 54A, 397-420. 
Plaza, M., Cohen, H., \& Chevrie-Muller, C. (2002). Oral language deficits in dyslexic children: Weaknesses in working memory and verbal planning. Brain and Cognition, 48(2-3), 505-512.

Protopapas, A., Archonti, A. \& Skaloumbakas, C. (2007). Reading ability is negatively related to Stroop interference. Cognitive Psychology, 54(3), 251-282.

Reiter, A., Tucha, O. \& Lange, K.W. (2005). Executive Functions in Children with Dyslexia. Dyslexia, 11, 116-131.

Schofield, N. J., \& Ashman, A. F. (1986). The relationship between digit span and cognitive processing across ability groups. Intelligence, 10, 59-73.

Seidman, L.J., Biederman, J., Weber, W., Hatch, M. \& Faraone, S.V. (1998). Neuropsychological function in adults with attention-deficit hyperactivity disorder. Biological Psychiatry, 44(4), 260-268.

Siegel, L. S., \& Ryan, E. B. (1989). The development of working memory in normally achieving and subtypes of learning disabled children. Child Development, 60, 973-980.

Smith, E.E., Jonides, J. \& Koeppe, R.A. (1996). Dissociating verbal and spatial working memory using PET. Cerebral Cortex, 11, 11-20.

Smith-Spark, J.H., \& Fawcett, A., Nicolson, R. \& Fisk, J.E., (2004). Dyslexic students have more everyday cognitive lapses. Memory, 12(2), 174 - 182. 
Smith-Spark, J. H., \& Fisk, J.E. (2007). Working memory functioning in developmental dyslexia. Memory, 15, 34-56.

Smith-Spark, J.H., Fisk, J.E., Fawcett, A \& Nicolson, R. (2003). Investigating the central executive in adult dyslexics: Evidence from phonological and visuospatial working memory performance. European Journal of Cognitive Psychology, 15(4), 567-587.

Smyth MM, Pearson NA., \& Pendleton LR. (1988). Movement and working memory: Patterns and positions in space. Quarterly Journal of Experimental Psychology, 40, 497 514.

Smyth MM., \& Pendleton LR. (1989). Working memory for movements. Quarterly Journal of Experimental Psychology, 41A, 235-250.

Smyth, M. M., \& Scholey, K. A. (1992). Determining spatial span: The role of movement time and articulation rate. Quarterly Journal of Experimental Psychology, 45A, 479-501.

Swanson, H. L. (1984). Semantic and visual memory codes in learning disabled readers. Journal of Experimental Child Psychology,37, 124-140.

Swanson, H. L. (1999). Strategy instruction: overview of principles and procedures for effective use. Learning Disability Quarterly, 12, 3-14. 
Swanson, H. L. (2003). Age-related differences in learning disabled and skilled readers' working memory. Journal of Experimental Child Psychology, 85, 1-31.

Swanson, H. L., Reffel, J., \& Trahan, M. (1991). Naturalistic memory in learning disabled and skilled readers. Journal of Abnormal Child Psychology, 19(2), 117-147.

Swanson, H. L., Ashbaker, M.,H. \& Lee, C. (1996). Working memory in learning disabled readers as a function of processing demands. Journal of Experimental Child Psychology, $61,242-275$.

Swanson, H. L., \& Howell, M. (2001). Working memory, short-term memory, and speech rate as predictors of children's reading performance at different ages. Journal of Educational Psychology, 93, 720-734.

Swanson, H. L. \& Jerman, O. (2007). The influence of working memory on reading growth in subgroups of children with reading disabilities. Journal of Experimental Child Psychology, 96, 249-283.

Swanson, H.L., Zheng, X \& Jerman, O. (2009). Working Memory, Short-Term Memory, and Reading Disabilities: A Selective Meta-Analysis of the literature. Journal of Learning Disabilities, 42(3), 260-287.

Tijms, J. (2004). Verbal memory and phonological processing in dyslexia. Journal of Research in Reading, 27(3), 300-310. 
Torgesen, J. K., \& Goldman, T. (1977). Rehearsal and short-term memory in second grade reading disabled children. Child Development, 48, 56-61.

Vail, P.L. (1990). Gifts, talents and the dyslexias: wellsprings, springboards and finding Foly's rocks. Annals of Dyslexia, 40, 3-17.

Vandierendonck, A., Kemps, E., Fastame, M.C. \& Szmalec, A. (2004). Working memory components of the Corsi blocks task. British Journal of Psychology, 95, 57-79.

Vandierendonck, A. \& Szmalec, A. (2004). An asymmetry in the visuospatial demands of forward \& backward recall in the Corsi blocks task. Imagination, Cognition and Personality $23(2-3), \quad 225-231$.

Vecci, T. \& Richardson, J.T.E. (2001). Measures of visuospatial short term memory: the knox cube imitation test and the Corsi blocks test compared. Brain and Cognition, 46, 291294.

Vellutino, F. R. (1979). Dyslexia: Theory and Research. Cambridge, MA: MIT Press.

Vellutino, F. R., Fletcher, J.M., Snowling, M.J. \& Scanlon, D.M. (2004). Specific reading disability (dyslexia): what have we learned in the past four decades. Journal of Child Psychology and Psychiatry, 45(1), 2-40. 
Wagner, R., \& Muse, A. (2006). Short-term memory deficits in developmental dyslexia. In T. Alloway, \& S. Gathercole (Eds.), Working Memory and Neurodevelopmental Disorders (pp. 41-57). New York, NY: Psychology Press.

Wechsler, D. (1993). The Wechsler Objective Reading Dimensions. London: The Psychological Corporation.

Wechsler, D. (1999). The Wechsler Abbreviated Scale of Intelligence (WASI). London: The Psychological Corporation.

West, T.G. (1997). In the Mind's Eye: Visual Thinkers, Gifted People with Dyslexia \& Other Learning Difficulties. New York: Prometheus.

West, T.G. (2008). Its time to get serious about the talents of dyslexics. IDA Perspectives: Summer Issue. International Dyslexia Association.

Wilde, N., Strauss, E., \& Tulsky, D. S. (2004). Memory span on the Wechsler scales. Journal of Clinical and Experimental Neuropsychology, 26, 539-549.

Willcutt, E.G., \& Pennington, B.F. (2000). Comorbidity of reading disability and attentiondeficit/hyperactivity disorder: Differences by gender and subtype. Journal of Learning Disabilities, 33, 179-191.

Wilson, B., Baddeley, A.D. \& Young, A.W. (1999). LE, a person who lost her "mind's eye". Neurocase, 5, 119-127. 
Wolf, R.C., Sambataro, F., Lohr, C., Steinbrink, C., Martin, C. \& Vasic, N. (2010). Functional brain network abnormalities during verbal working memory performance in adolescents and young adults with dyslexia. Neuropsychologia, 48, 309-318.

Wong, B. (1978). The effects of directive cues on the organization of memory and recall in good and poor readers. Journal of Educational Research, 72, 32-38. 


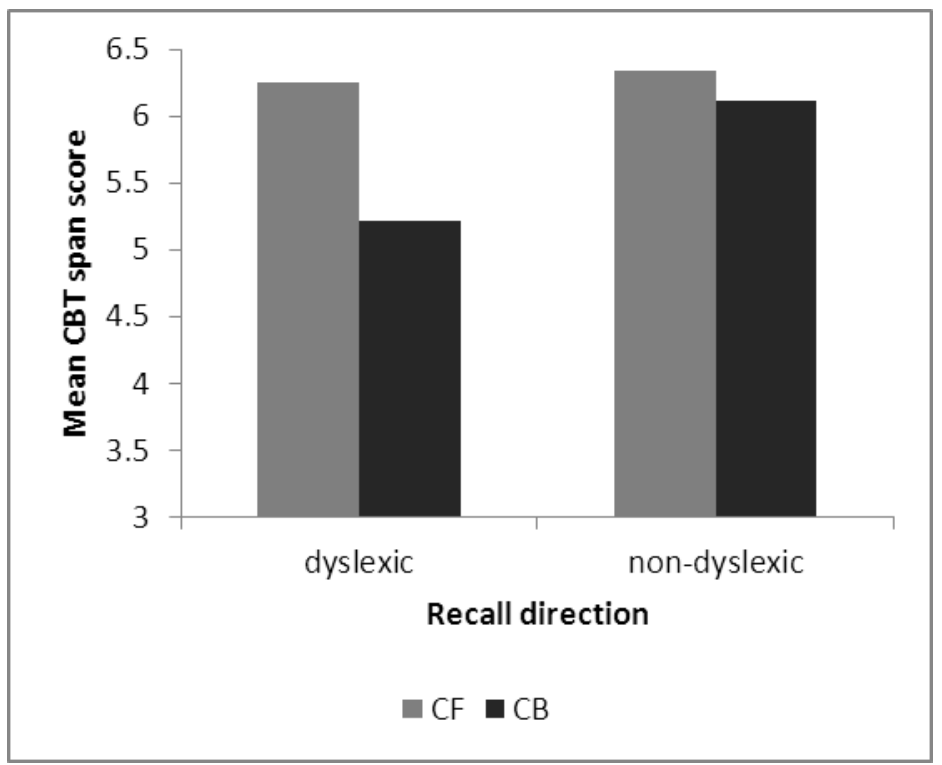

Figure 1. Experiment 1: Mean CBT span scores for dyslexic and non-dyslexic participants on $\mathrm{CF}$ and $\mathrm{CB}$. 


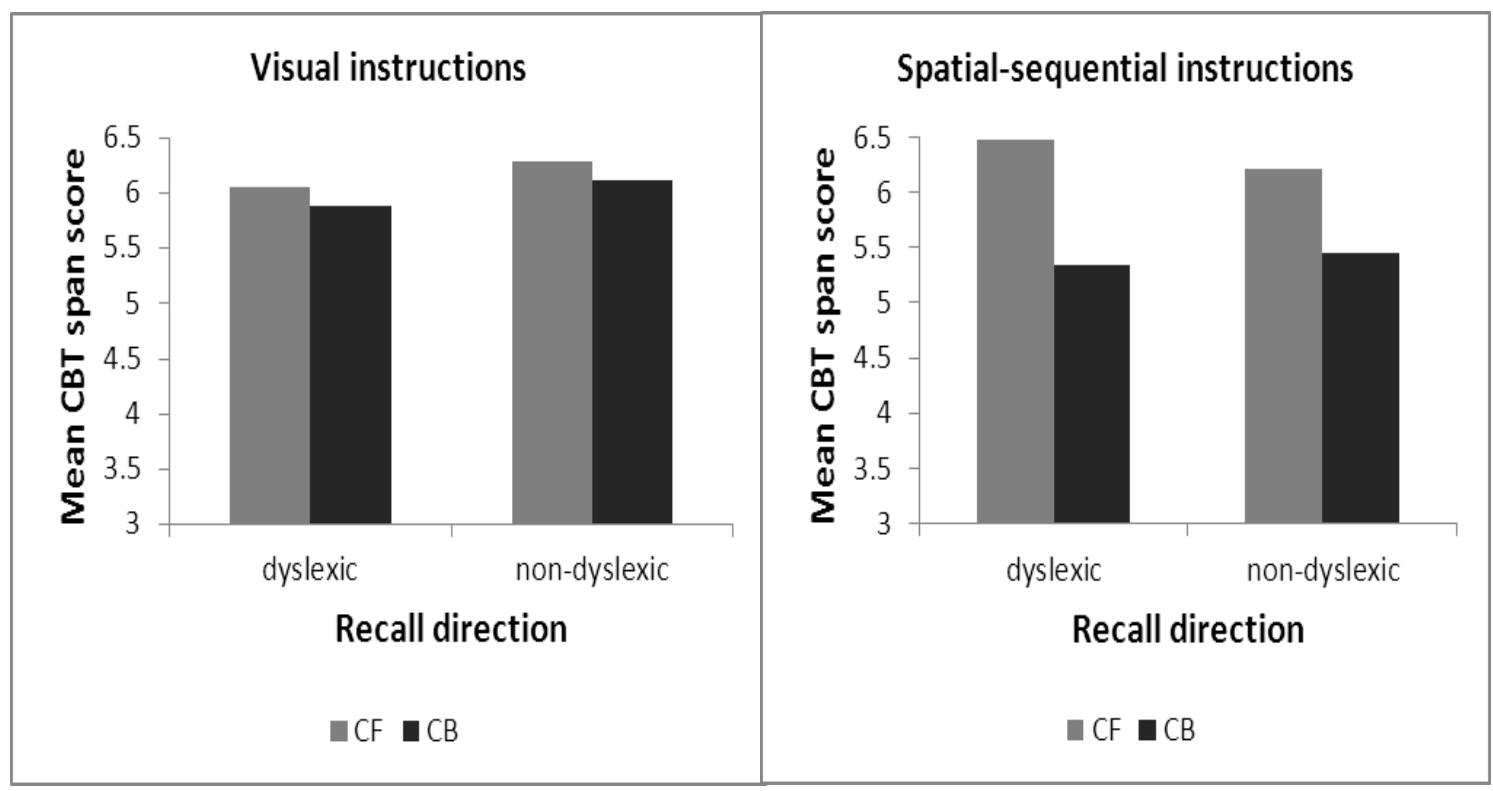

Figure 2. Experiment 2: CF and CB span scores under visual and sequential instruction conditions. 


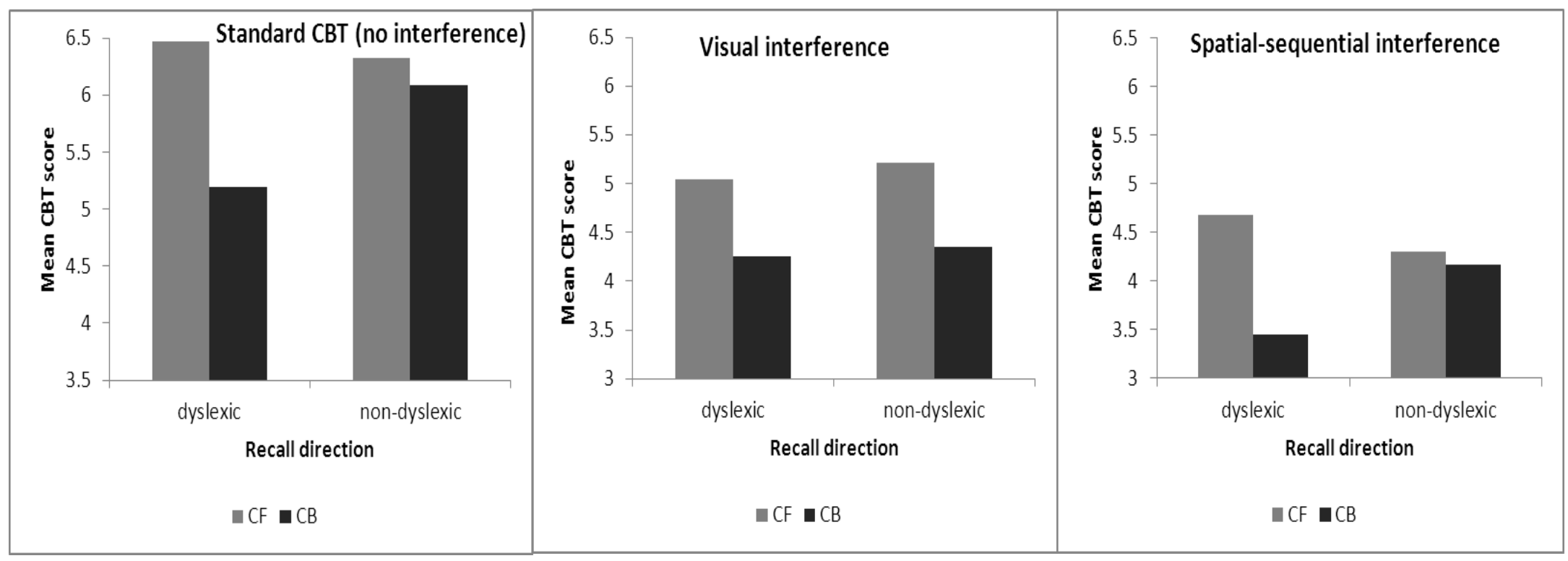

Figure 3. Experiment 3: CF and CB span scores under standard (no interference), visual and sequential interference conditions. 
Table1. Experiment 3: correlations between VPT score and CF/CB span scores in all three interference conditions

\begin{tabular}{lcccccc} 
& \multicolumn{2}{c}{ No interference } & \multicolumn{2}{c}{ Visual interference } & \multicolumn{2}{c}{ Spatial interference } \\
& $\mathbf{C F}$ & $\mathbf{C B}$ & $\mathbf{C F}$ & $\mathbf{C B}$ & $\mathbf{C F}$ & $\mathbf{C B}$ \\
\hline Dyslexic & .06 & -.13 & .12 & -.11 & .17 & -.06 \\
Non-dyslexic & -.12 & $.60^{* *}$ & .03 & .09 & $.38^{*}$ & $.44^{* *}$ \\
& & & & & & \\
$\begin{array}{l}\text { Between group } \\
\text { comparison }\end{array}$ & $\mathrm{ns}$ & $\begin{array}{l}Z=3.32 \\
p<.001\end{array}$ & $\mathrm{~ns}$ & $\mathrm{~ns}$ & $\begin{array}{c}Z=.92 \\
p=.18\end{array}$ & $\begin{array}{c}Z=2.14 \\
p=.02\end{array}$ \\
\hline
\end{tabular}

\footnotetext{
* Correlation sig. at .05 level

** Correlation sig. at .01 level
} 


\section{APPENDIX A}

Dyslexic and non-dyslexic participants compared on age and screening test scores for all three experiments. In each case, all WASI IQ measures are age-corrected T-scores. WORD and DAST NWR are raw scores.

\begin{tabular}{|c|c|c|c|c|c|}
\hline & \multicolumn{2}{|c|}{ Dyslexic } & \multicolumn{2}{|c|}{ Non-dyslexic } & \multirow{2}{*}{$\begin{array}{c}\text { Between } \\
\text { group } \\
\text { p-value }\end{array}$} \\
\hline & mean & sd & mean & sd & \\
\hline \multicolumn{6}{|l|}{ Experiment 1} \\
\hline Age in years & 29.56 & 11.95 & 27.10 & 10.78 & .21 \\
\hline WASI VIQ & 104.09 & 13.28 & 106.91 & 12.40 & .20 \\
\hline WASI PIQ & 114.34 & 11.91 & 112.41 & 15.87 & .41 \\
\hline WASI FSIQ & 110.24 & 10.78 & 111.39 & 10.55 & .53 \\
\hline WORD spelling $(\max 50)$ & 40.46 & 3.00 & 45.27 & 3.60 & $<.001$ \\
\hline DAST NWR (max 99) & 76.88 & 8.02 & 92.05 & 6.61 & $<.001$ \\
\hline \multicolumn{6}{|l|}{ Experiment 2} \\
\hline Age in years & 25.9 & 9.6 & 27.6 & 11.4 & .45 \\
\hline WASI VIQ & 103.6 & 13.2 & 104.6 & 12.1 & .71 \\
\hline WASI PIQ & 115 & 11.0 & 112.1 & 9.4 & .21 \\
\hline WASI FSIQ & 110.3 & 10.9 & 109.3 & 10.4 & .69 \\
\hline WORD spelling $(\max 50)$ & 40.3 & 3.4 & 46.0 & 3.6 & $<.001$ \\
\hline DAST NWR (max 99) & 75.5 & 11.4 & 94.0 & 6.1 & $<.001$ \\
\hline \multicolumn{6}{|l|}{ Experiment 3} \\
\hline Age in years & 23.6 & 5.9 & 23.4 & 8.5 & .88 \\
\hline WASI VIQ & 96.1 & 8.2 & 97.3 & 10.0 & .52 \\
\hline WASI PIQ & 112.5 & 8.9 & 109.0 & 11.0 & .13 \\
\hline WASI FSIQ & 104.5 & 6.0 & 103.1 & 9.8 & .44 \\
\hline WORD spelling ( $\max 50)$ & 39.6 & 7.9 & 45.3 & 3.0 & $<.001$ \\
\hline DAST NWR (max 99) & 70.9 & 10.3 & 92.4 & 6.3 & $<.001$ \\
\hline
\end{tabular}




\section{Footnote 1}

A 2 group (dyslexic; non-dyslexic) x 2 recall direction (CF; CB) x 3 interference type (none; visual; spatial) ANOVA presented significant main effects of interference type, $F(2,138)=$ 78.57, $M S E=1.67, p<.001, \eta^{2}=.53$ and recall direction, $F(1,69)=57.12, M S E=.98, p<$ $.001, \eta^{2}=.45$. Bonferroni post-hoc tests illustrated that, taken overall, recall on CF $(M=$ $5.32, S D=.11)$ was superior to that on CB $(M=4.60, S D=.10)$ and was highest in the no interference condition $(M=6.03, S D=.08)$ compared to both visual $(M=4.69, S D=.14)$ and spatial $(M=4.16 ; S D=3.84)$ conditions $(p<.001$ in both cases). Recall was also significantly higher with visual compared to spatial interference $(p=.004)$. A significant group*direction interaction, $F(1,69)=12.60, M S E=.98, p=.001, \eta^{2}=.15$, showed that, overall, the CF superiority effect was stronger for dyslexics (CF $M=5.36, S D=.17$; CB $M$ $\left.=4.30, S D=.14 ; p<.001, \eta^{2}=.60\right)$ than non-dyslexics $(\mathrm{CF} M=5.28, S D=.16$; $\mathrm{CB} M=$ $\left.4.90, S D=.13 ; p=.003, \eta^{2}=.22\right)$.

A significant 3 way interaction: $F(2,138)=7.33, M S E=.69, p=.001, \eta^{2}=.10$, represents the between-group differences illustrated in Figure 3. Dyslexics perform less well on CB compared to $\mathrm{CF}$ in all 3 conditions, whereas for non-dyslexics this only occurs with visual interference. Means relevant to this interaction are shown in the main analyses above. 